\title{
A Novel Approach to DG Curtailment in Rural Distribution Networks - A Case Study of the Avacon Grid as Part of the InterFlex Field Trial
}

\author{
Thorsten Gross, Sven Reese, Benjamin Petters \\ Avacon Netz GmbH \\ Salzgitter, Germany \\ \{thorsten.gross, sven.reese, benjamin-georg.petters\}@avacon.de
}

\author{
Marco Cupelli, Dominik Mildt, Antonello Monti \\ Institute for Automation of Complex Power Systems \\ E.ON Energy Research Center RWTH Aachen University \\ Aachen, Germany \\ \{mcupelli,dmildt, amonti\}@eonerc.rwth-aachen.de
}

\begin{abstract}
Distribution system operators in rural areas of Germany are frequently facing imminent equipment overloading caused by the feed-in of local renewable Distributed Generation (DG). A grid operator's last resort to maintain system stability and avoid protection tripping is to temporarily curtail local feed-in until hosting capacity in the network has caught up with the demand. Due to technological limitations in today's networks the volume of curtailed energy can be greater than what would strictly be necessary. This paper presents a case study of a $110 \mathrm{kV}$ overhead line in Avacon's network and demonstrates the limitations of today's approach to DG curtailments, especially the relative coarse granularity of control steps. The authors develop a novel control algorithm for emergency curtailments that takes advantage of technological improvements and describes the architecture for a successful deployment at the example of Avacon's network and SCADA. The authors compare the amount of curtailed energy under today's best practice with the theoretical optimum and the novel approach.
\end{abstract}

Keywords-Generation curtailment, distribution network, renewable energy sources, optimization, distributed generation, thermal overloading

\section{INTRODUCTION}

Distribution System Operators (DSOs) in Germany with a large share of installed DG and long feeders in their networks experience frequent voltage limit violations and the risk of equipment overload in their networks [1]. Moreover, congestion is becoming a critical issue in densely clustered grids network [2]. Therefore, innovative grid congestion management strategies are needed to enable the DSO to deal with the new energy landscape [3]. Consequently, the topic of congestion management and curtailment lately received notable attention in research [4]-[10].

Over the past decade Germany has seen a significant growth in decentralized renewable energy sources (RES). According to the renewable energy act grid operators have a legal obligation to connect all DGs to their network and accommodate all energy that is being produced by renewable DGs [11]. To comply with this regulation, the challenges that German DSOs are facing are twofold: First, the DGs are often located in rural areas where the hosting capacity of the network is not traditionally designed to deal with their presence. Second, the volatility and unpredictability of RES puts additional operational burden on the DSO.

If the DGs' feed-in exceed the network's nominal capacity, there exists the risk of violating voltage limits or the equipment's thermal limits. In such situations, the grid operators have the option to temporarily curtail local feed-in to maintain system stability and avoid protection tripping. Curtailment options of DG come with the obligation to increase the network's hosting capacity as soon as possible [12]. However, grid operators had difficulties catching up with the growth of renewable energy in recent years, which resulted in a total annual cost for curtailment actions of $373 \mathrm{M} €$ in 2016 [13].

For many grid operators it is best practice to control small scale DGs via long wave radio signals. With these signals grid operators can limit the generators output to $100 \%, 60 \%, 30 \%$ or $0 \%$ of its nominal power. While this technology has proven to be simple, robust and cost-effective it also comes with several drawbacks:

1) The lack of communication backchannel, making it impossible to confirm whether the signal has been received and acted upon.

2) The limitation of only four discrete setpoints.

3) The limited number of receiver assignments, as in many areas the DGs are often placed in medium voltage and low voltage networks, where they are clustered under one radio frequency. The motivation for this approach was that on the one hand earlier SCADA could not automate the process of receiver assignment. On the other hand, it was OPEX driven as the service provider charges per signal address.

The combination of 2) and 3) means that in practice curtailment actions can only be carried out in comparably large discrete steps. As a result, it is very difficult to adjust the output precisely to the required technical limits and oftentimes DSOs are forced to curtail more energy than theoretically needed. Hence, a novel approach for DG curtailment, which features finer granularity and dedicated bi-directional communication channels is required, leveraging on the advancements in data transmission and communication technology.

(c) 2018 IEEE. Personal use of this material is permitted.

Permission from IEEE must be obtained for all other users.

DOI: 10.1109/INDIN.2018.8472095.

Publisher version: https://ieeexplore.ieee.org/document/8472095 
This paper presents the Smart Grid Hub (SGH), which is developed by Avacon as part of the H2020 InterFlex Project [15]. The SGH specifically target these challenges, making use of the widespread smart meter infrastructure rollout in Germany in combination with the increased data processing capabilities of latest supervisory control and data acquisition (SCADA) systems. A case study proposes its application to assist DSOs with an optimized curtailment strategy that enables an increasing share of RES in the grid, which could not be achieved with the best practice approach.

The remainder of this paper is organized as follows. Section II briefly explains the SGH architecture and how it integrates with existing SCADA and other legacy systems. Section III then introduces the best practice approach to curtailment, as well as a novel algorithm that makes use of the increased communication capabilities. Section IV presents one of Avacons's most frequently congested high voltage (HV) systems as a case study for simulation. Section V shows some simulation results and compares both approaches to curtailment. Finally, section VI gives a short conclusion.

\section{The SMART GRID Hub}

The SGH acts as an extension of the distribution network SCADA and - in combination with smart meters and next generation signal receivers that connect to the smart meter gateway - allows grid operation engineers on duty to control small scale DG individually. As a result, the SGH enables advanced curtailment algorithms that respect the limitations of day-to-day grid operation while taking advantage of advanced control options. For instance, automatic control of a large number of small scale DG can be fully automated to require only minimal input from the grid operation engineer.

In order to comply with the unbundling regulations and obligations derived from the renewable energy act, the SGH approach has been developed and is currently implemented and tested within the InterFlex project. The SGH approach consists of a grid management system, which acts as an aggregation/disaggregation platform interfacing the DSO SCADA system with the residential smart meter infrastructure.

The SGH depicted in Fig. 1 is located within the DSO SCADA environment and connects to it via a IEC60870-6 TASE. 2 interface. Outbound, it has interfaces to the highly secured public smart meter gateway administration service, which provides access to smart meters in private households. The smart meter gateway administration and integration is defined in the technical guideline TR-03109-01 and regulated by the federal agency for cyber security, Bundesamt für Sicherheit in der Informationstechnik (BSI). Currently, three communication possibilities between SGH and smart meter gateways are supported:

\section{1) Communication via webservices \\ 2) Communication via LTE \\ 3) Communication via powerline}

The core functionality of the $\mathrm{SGH}$ is to disaggregate a single curtailment request from the grid operation engineer in charge into a set of individual control signals actuated on the DGs. It

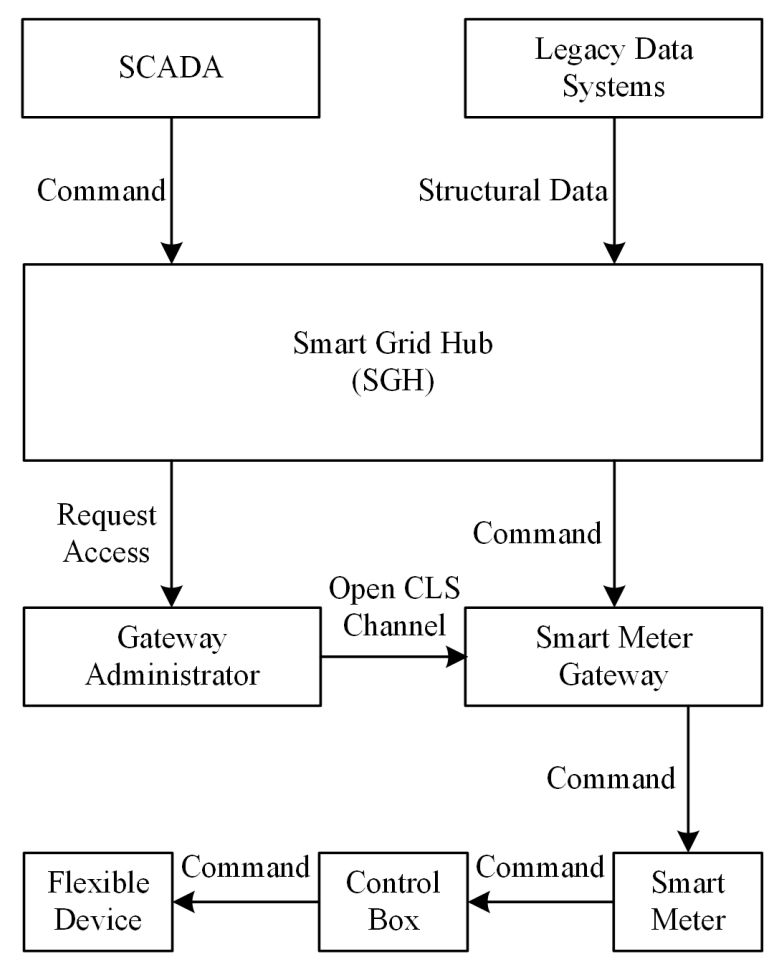

Figure 1: SGH Architecture and Flow of Commands

features an optimization engine, which determines the optimal set of control signals and handles the communication to the DGs in the field. The optimization engine enables dynamic clustering based on location, connection, or type of DG. A key benefit from the DSO perspective in this modular approach is that there is no need to alter the mission critical SCADA system to provide the desired additional functionality.

\section{DG CURTAILMENT IN CRITICAL SITUATIONS}

As mentioned, DG capacity and generation may vastly exceed demand in a region, to the point where the capacity limits of the power lines would be exceeded. This logically leads to the situation that the grid operator is forced to curtail the regional feed-in on several occasions to avoid equipment overload and tripping of protections. The operator has to determine the curtailment schedule while taking the following three criteria into account:

1) Ensuring safe operation of all equipment within technical limits at all times.

2) Limiting curtailments to a practical minimum.

3) Ensuring that all curtailment actions are carried out in a non-discriminatory manner to guarantee equal treatment of all customers.

Rural areas present themselves to be specifically challenging, as their spatial expansion allows a multitude of RES connected to a comparatively weak superordinate grid system, that is tasked with exporting excess power. This paper assumes a HV system to which encompasses several wind farms (WFs) are either directly connected to the HV lines or bundled at the medium voltage (MV) level and then connected via a HV/MV-substation. 
Other DGs include photovoltaics (PVs) and combined heat and power plants (CHPs), which are of a noticeably smaller scale. These are located at lower voltage levels, together with the system's loads and collectively connected to the HV system via $\mathrm{HV} / \mathrm{MV}$-substations. The following additional assumptions are made for the system:

- Generators only contribute active power to the line load.

- A single line or a combination of lines that share the load perfectly symmetrical is regarded, which allows to plan for a single overall capacity limit.

- Large scale WFs can accept four setpoints, limiting their output $100 \%, 60 \%, 30 \%$ or $0 \%$ of their nominal power. Small scale PVs and CHPs can accept one of two signals at a time, on or off.

- All generators can accept one control signal at the beginning of each 15-minute registration period.

\section{A. Best Practice Approach}

Based on the regulatory framework the DSOs in Germany are fully unbundled from all generation assets, while private companies usually operate DGs. The options to control these units however are limited. Large generation plants such as WFs are connected directly to the DSO SCADA, whereas most smaller units, like PVs and CHPs, are oftentimes only equipped with a long wave radio receiver, which are usually clustered per substation. Therefore, in best practice, control can only be exerted over the larger scale WFs. Historically, these units can accept setpoints to limit their momentary output to $100 \%, 60 \%$, $30 \%$ or $0 \%$ of their nominal power. When considering $i \in \mathcal{N}_{W F}$ WFs, where $\mathcal{N}_{W F}$ is the set of all WFs in the system, their curtailed power is given as:

$$
\begin{gathered}
P_{W F, \text { cur }}^{i}=\left\{\begin{array}{cr}
0 & \text { no curtailment } \\
P_{W F, a c t}^{i}-\alpha^{i} \cdot P_{W F, N}^{i} & \text { curtailment }
\end{array},\right. \\
\alpha^{i} \in\{0,0.3,0.6,1\} .
\end{gathered}
$$

Here, $P_{W F, \text { cur }}^{i}$ is the curtailed of $\mathrm{WF} i, P_{W F, a c t}^{i}$ is its momentary uncurtailed generation, $P_{W F, N}^{i}$ is its nominal power and $\alpha^{i}$ defines the actual setpoint. This limits a grid operator to a few discrete steps to curtail the DG feed-in. Consequently, this can result in an over-curtailment of regional DG production with the intention of avoiding equipment overload. The best practice curtailment algorithm can be stated as an optimization problem that aims to minimize the curtailed power.

$$
\begin{array}{ll}
\min & \sum_{i \in \mathcal{N}_{W F}} P_{W F, \text { cur }}^{i} \\
\text { s.t. } & (1), \\
& \sum_{i \in \mathcal{N}_{W F}}\left(P_{W F, \text { act }}^{i}-P_{W F, \text { cur }}^{i}\right) \\
& +\sum_{j \in \mathcal{N}_{P V}} P_{P V, \text { act }}^{j} \\
& +\sum_{k \in \mathcal{N}_{C H P}} P_{C H P, \text { act }}^{k} \\
& -P_{\text {Load }} \leq P_{\text {Line }, N} .
\end{array}
$$

The nominal line capacity is given by $P_{\text {Line,N }}$, while $P_{P V, \text { act }}^{j}$ states the uncurtailed generation of the PV generator $j \in \mathcal{N}_{P V}$, where $\mathcal{N}_{P V}$ is the set of all connected PV generators. Similarly, $P_{C H P, a c t}^{k}$ is the uncurtailed generation of the CHP $k \in \mathcal{N}_{C H P}$ and $\mathcal{N}_{C H P}$ is the set of all connected CHPs. Finally, $P_{\text {Load }}$ is the summed-up system load.

\section{B. SGH with fine granularity control}

The SGH enables the DSO to leverage the smart meter infrastructure, which will become standard in Germany in the upcoming years. This enables the possibility to control small DG individually and directly, which did not exist up to now. Consequently, the DSO will have a substantial number of additional options to shape the curtailment action and track the technical limits of stressed equipment more closely. The operating engineers can control smaller units, increasing the granularity and therefore reducing the overcompensation of the curtailment.

As stated previously, small scale PV and CHP applications can only be regulated to an on or off state. Their respective curtailed powers $P_{P V, c u r}^{j}$ and $P_{C H P, c u r}^{k}$ are therefore given by:

$$
\begin{gathered}
P_{P V, \text { cur }}^{j}=\beta^{j} \cdot P_{P V, a c t}^{j}, \\
\beta^{j} \in\{0,1\}, \\
P_{C H P, \text { cur }}^{k}=\gamma^{k} \cdot P_{C H P, a c t}^{k}, \\
\gamma^{k} \in\{0,1\} .
\end{gathered}
$$

The setpoints are defined by the binary indicators $\beta^{j}$ and $\gamma^{k}$. Again, an optimization problem can be formulated to minimize the curtailed power:

$$
\begin{array}{ll}
\min & \sum_{i \in \mathcal{N}_{W F}} P_{W F, c u r}^{i}+\sum_{j \in \mathcal{N}_{P V}} P_{P V, \text { cur }}^{j}+ \\
& +\sum_{k \in \mathcal{N}_{C H P}} P_{C H P, \text { cur }}^{k} \\
\text { s.t. } \quad & (1),(3),(4) \\
& \sum_{i \in \mathcal{N}_{W F}}\left(P_{W F, a c t}^{i}-P_{W F, \text { cur }}^{i}\right) \\
& +\sum_{j \in \mathcal{N}_{P V}}\left(P_{P V, \text { act }}^{j}-P_{P V, \text { cur }}^{j}\right) \\
& +\sum_{k \in \mathcal{N}_{C H P}}\left(P_{C H P, \text { act }}^{k}-P_{C H P, \text { cur }}^{k}\right) \\
& -P_{\text {Load }} \leq P_{\text {Line,N }} .
\end{array}
$$

\section{Testcase System}

The focus of the case study is the system depicted in Fig. 2, which consists of two $110 \mathrm{kV}$ overhead lines in northern Germany. The considered HV lines connect two small cities over a distance of $29 \mathrm{~km}$. Each line system is equipped with three phase $\mathrm{Al} / \mathrm{St}$ conductors of the type $1 * 3 * 1 * 150 / 25 \mathrm{~mm}^{2} \mathrm{Al} / \mathrm{St}$. At standard conditions, the conductor is rated for $465 \mathrm{~A}$, which corresponds to $90 \mathrm{MW}$ of total transmission capacity per system at $110 \mathrm{kV}$. As stated previously, HV lines are treated as a single system with the summed capacity of the single conductors. This results in a thermal limit of $930 \mathrm{~A}$ or $P_{\text {Line }, N} \cong 180 \mathrm{MW}$ at standard operating conditions. 


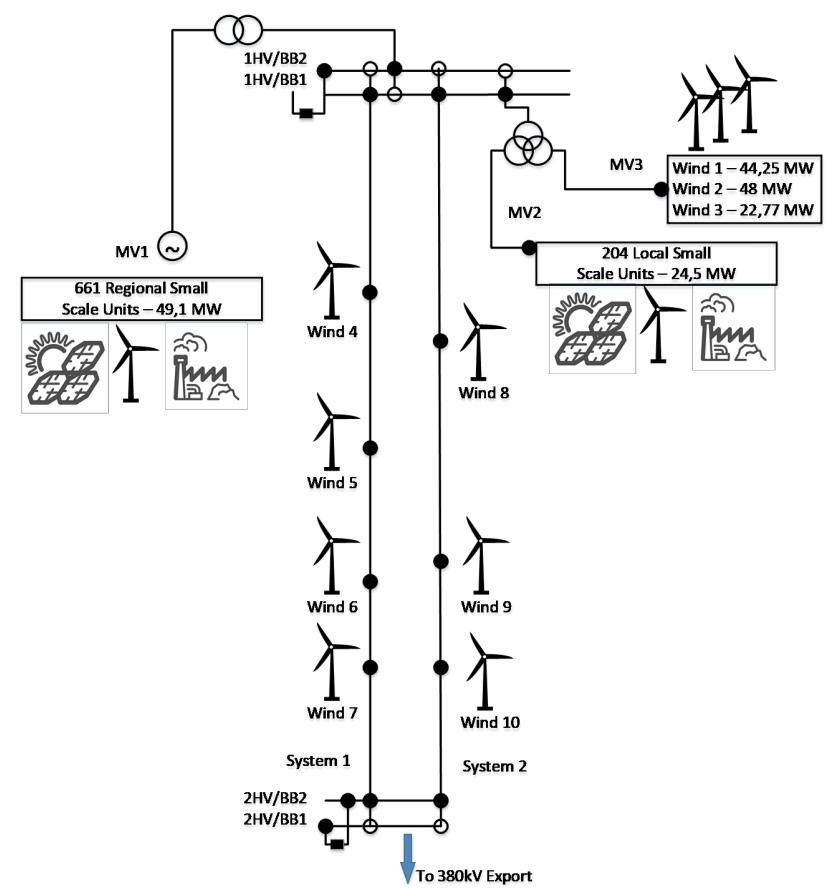

Figure 2: Grid Section

Currently, a total of 7 windfarms are connected directly to the $110 \mathrm{kV}$ lines, where four of those are connected to system 1 and the other three are connected to system 2. Another three windfarms are connected at lower voltage levels. The total nominal power of these windfarms amounts to $321.4 \mathrm{MW}$. Additionally, a total of $73 \mathrm{MW}$ of small scale DGs are installed in the underlying MV and low voltage (LV) networks. DSO operation is challenged by the fact that the total installed capacity of DGs amounts to almost $400 \mathrm{MW}$, which is more than twice the amount of power that the line can transport. At the same time, the annual net peak load is approximately $80 \mathrm{MW}$. This corresponds to only $20.26 \%$ of installed DG and currently leaves curtailment as the only option in case of high RES generation.

To underline the necessity of analyzing and improving curtailment behavior, Fig. 3 and 4 show historical data of the curtailment requests within 2016 and 2017 for the example system. Fig. 3 depicts the history of curtailed power magnitude, while Fig. 4 illustrates the duration of the requests, sorted descending from large to small values respectively. Clearly, the previous hold true, as within these two years significant curtailment efforts were made. In total, the network operated under curtailment for more than 26300 min, which corresponds to about $2.5 \%$ of the whole timeframe.

In our example, a week in summer 2017 is analyzed. A combination of high wind production and low consumption lead to imminent equipment overload at several occasions during this week. Power output time series for small scale generators are represented by synthetical data, multiplying their nominal power with a reference time series. The reference time series for $\mathrm{PV}$ is the PV forecast of regional transmission system operator (TSO), 50 Hertz Transmission. The reference time series for single wind generators is the normalized time series of one the regional windfarms. CHPs are assumed to run at full capacity all the time.

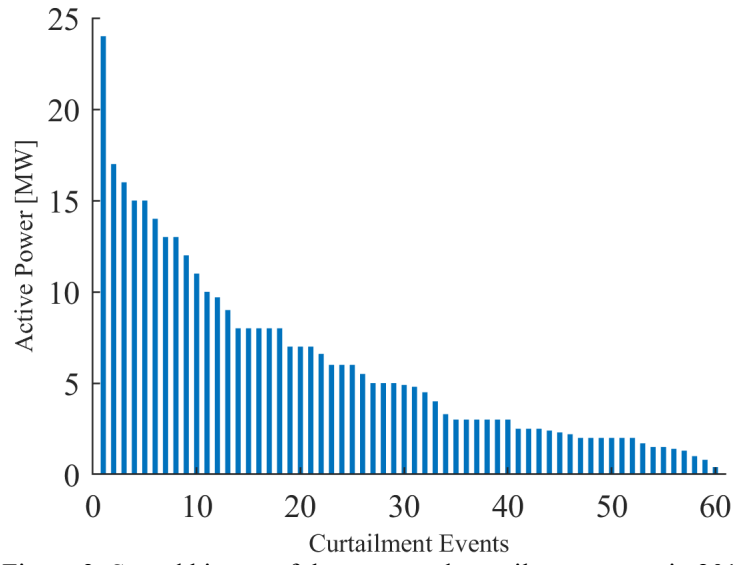

Figure 3: Sorted history of the requested curtailment power in 2016/17

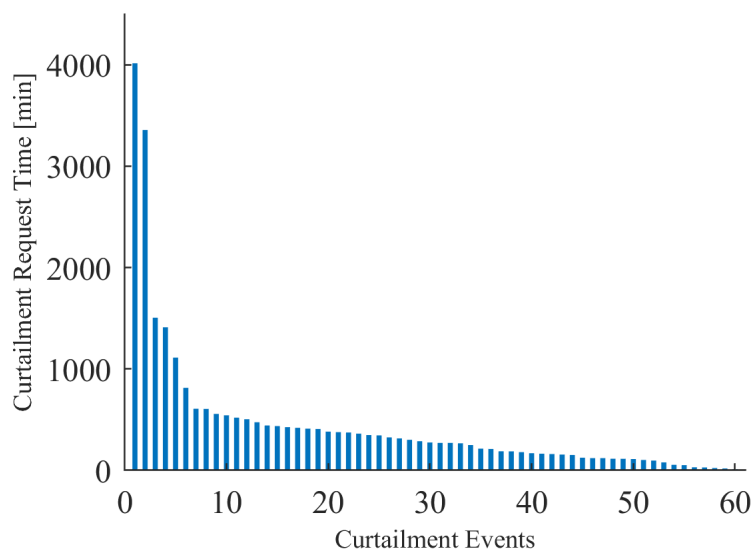

Figure 4: Sorted history of the curtailment request durations in 2016/17

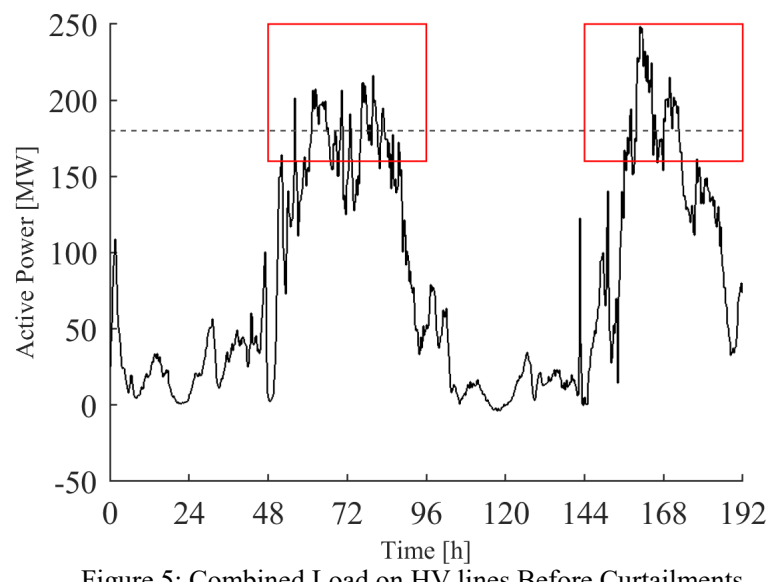

Fig. 5 shows the combined load on the two HV-lines. Positive values correspond to net power exports and negative values to net power imports. During the observed interval several curtailments were carried out at the request of the connected TSO. An interpolation between the last value before the curtailment action took place and the first value after the end of the curtailment action was performed to show the hypothetical overhead line utilization without external interference, i.e. 


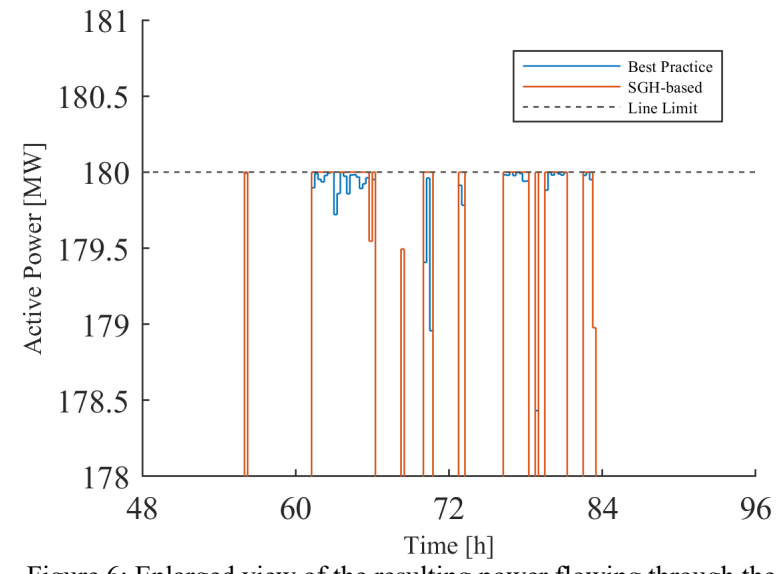

Figure 6: Enlarged view of the resulting power flowing through the HV line for the first period highlighted in Fig. 5

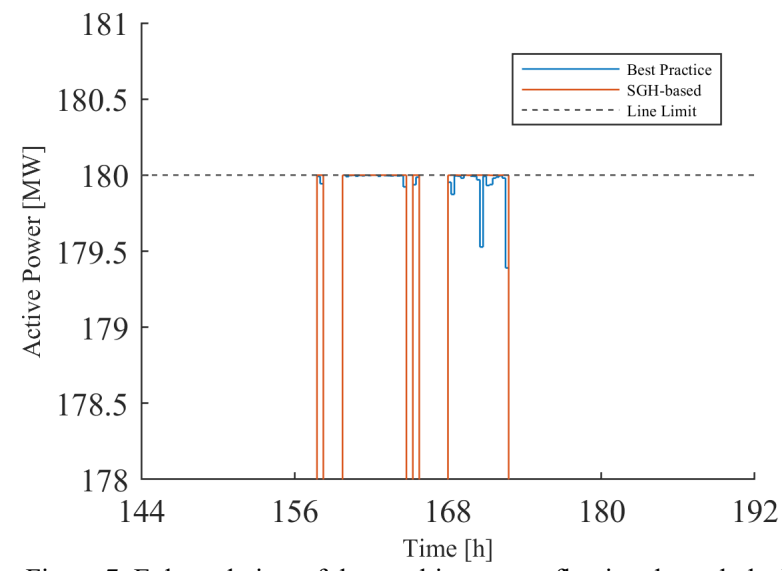

Figure 7: Enlarged view of the resulting power flowing through the HV line for the second period highlighted in Fig. 5

curtailment requests. The grey line indicates the maximum line capacity and the red boxes highlight time periods during which significant curtailment was needed.

Without curtailment, the static thermal limit of the conductor would have been violated for a total 1305 mins which correspond 87 of the 15 -minute registration periods in the regarded week. The maximum line overload would have amounted to $68.285 \mathrm{MW}$ while the average power overload would have been 21.702 MW. Total surplus energy beyond the conductors' capacity would have amounted to 472.009 MWh. This also represents the lower boundary for the curtailed energy.

\section{Testcase Simulation}

The test system was modeled in MATLAB [16] to compare best practice and SGH-based curtailment for the presented week. Optimization problems were created using the free YALMIP toolbox [17], using a mixed integer formulation to map binary and logic constraints in (1), (3) and (4). The commercial Gurobi solver [18] was employed in the actual calculation. As explained previously, todays best practice only made use of the flexibility from large windfarms and limiting the available setpoints to four discrete steps of $100 \%, 60 \%, 30 \%$ and $0 \%$ of nominal power. In the SGH-based approach small scale generator control was

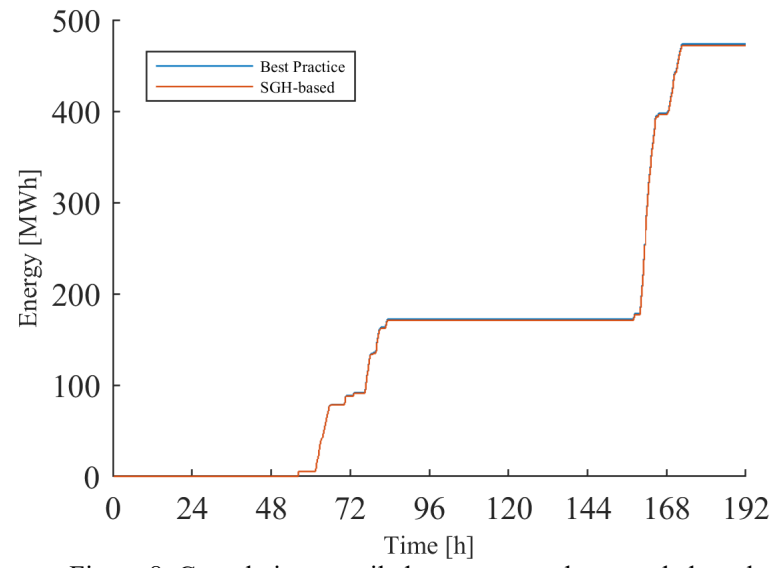

Figure 8: Cumulative curtailed energy over the regarded week

TABLE I. OVERVIEW OF CURTAILMENT EVENTS

\begin{tabular}{|l|l|l|l|l|}
\hline \multirow{2}{*}{} & \multicolumn{4}{|c|}{ Best practice } \\
\cline { 2 - 5 } & \multicolumn{1}{|c|}{$\boldsymbol{\operatorname { m i n }}$} & $\boldsymbol{\operatorname { m a x }}$ & $\boldsymbol{\varnothing}$ & $\Sigma$ \\
\hline Power [MW] & 0.413 & 68.292 & 21.781 & - \\
\hline $\begin{array}{l}\text { Energy } \\
{[\mathrm{MWh}]}\end{array}$ & 0.103 & 17.073 & 5.446 & 473.755 \\
\hline & \multicolumn{5}{|c|}{ SGH-based } \\
\cline { 2 - 5 } & $\boldsymbol{\operatorname { m i n }}$ & $\max$ & $\boldsymbol{\varnothing}$ & $\Sigma$ \\
\hline Power [MW] & 0.400 & 68.285 & 21.702 & - \\
\hline $\begin{array}{l}\text { Energy } \\
{[\mathrm{MWh}]}\end{array}$ & 0.100 & 17.071 & 5.425 & 472.016 \\
\hline
\end{tabular}

introduced to increase the granularity of curtailment actions. Tab. I provides indicators of the resulting power and energy curtailment.

Fig. 6 and 7 show an enlarged view of the active power exported during the highlighted regions of Fig. 5 under the two different curtailment algorithms. Evidently, the finer granularity of the SGH-based approach allows operation closer to the actual conductor capacity limit in many cases. In most cases, the remaining deviation from the conductor limit becomes indiscernible on the MW scale. Fig. 8 additionally shows the propagation of curtailed energy by its cumulative amount over the past time. Although differences seem minor, the best practice approach consistently required curtailment of additional energy.

Including the smaller generators in the set of potential curtailments expanded the space of possible actions and hence increased the granularity of curtailment commands. As a result, the grid's ability to accommodate local generation could be increased and the amount of curtailed energy required to ensure a safe and secure operation of the grid was reduced. The new approach reduced the excess curtailments to virtually zero, saving $1.739 \mathrm{MWh}$ or $3.67 \%$ of the energy in this scenario.

Analysis showed that the best practice approach to DG curtailments enabled the DSO to reach a solution which was very close to the technical optimum, at 100,002 \% of the lower boundary of required curtailments. This worse-than-optimal solution was caused by the small modulation option. We may recall that the grid operator had to achieve the target value on curtailments being able to control a limited number of DGs and with only a small number of discrete setpoints. 


\section{CONCLUSION}

In the light of the sheer amount of annual curtailments in Germany, every $\mathrm{kWh}$ reduction in curtailments can be considered a success, especially since public understanding and approval for these actions is very limited. Extrapolating the findings of our analysis across all grid operators in Germany, the new approach could have saved $137 \mathrm{GWh}$ in curtailments and almost 13,7 M€ in reimbursements in 2016 alone.

This motivates further work to better quantify the relationship between savings in curtailments and the number of DGs, the number of discrete power setpoints and the energy source of the DGs, as well as to further eliminate the errors introduced by using reference timeseries for the smaller scale generators. For future analysis, it might also prove interesting to investigate the impact of shorter intervals of control actuated, possibly taking into account coupling constraints over several time steps. The large WFs hardly react faster than within a 15-minute timeframe, but small scale DGs could realistically react to control signals within a much shorter timeframe.

Beyond the technical questions a deeper analysis of the economics of the presented approach seems relevant. While the availability of a communication channel via the smart meter framework can be assumed a given in the future in Germany, the required CAPEX and costs for operation and maintenance of such a system can offset a share of the savings this paper has demonstrated.

\section{ACKNOWLEDGMENT}

We wish to acknowledge the contributions of all experts involved in the project InterFlex (Grant No: 731289) co-funded by the European Commission as part of the H2020 Program (H2020-LCE-2016-SGS). Website: http://interflex-h2020.com/

\section{REFERENCES}

[1] A. N. M. M. Haque, M. T. Rahman, P. H. Nguyen and F. W. Bliek, "Smart curtailment for congestion management in LV distribution network," 2016 IEEE Power and Energy Society General Meeting (PESGM), Boston, MA, 2016, pp. 1-5.

[2] A. N. M. M. Haque, T. H. Vo and P. H. Nguyen, "Distributed intelligence: Unleashing flexibilities for congestion management in smart distribution networks (Invited paper)," 2016 IEEE International Conference on Sustainable Energy Technologies (ICSET), Hanoi, 2016, pp. 407-413.
[3] R. A. Verzijlbergh, L. J. De Vries and Z. Lukszo, "Renewable Energy Sources and Responsive Demand. Do We Need Congestion Management in the Distribution Grid?," in IEEE Transactions on Power Systems, vol. 29, no. 5, pp. 2119-2128, Sept. 2014.

[4] M. Andrychowicz and B. Olek, "Energy storing vs. generation curtailment - The measures for controlling renewable generation," 2017 14th International Conference on the European Energy Market (EEM), Dresden, 2017, pp. 1-5.

[5] R. L. Taljaard, M. R. Hammond, G. W. Ault, R. MacDonald, E. Davidson and P. Almeida, "Standardisation of curtailment analysis and the implications for distribution network operators and generators," CIRED Workshop 2016, Helsinki, 2016, pp. 1-4.

[6] V. Mohan, M. P. R. Suresh, J. G. Singh, W. Ongsakul and B. K. Kumar, "Online optimal power management considering electric vehicles, load curtailment and grid trade in a microgrid energy market," 2015 IEEE Innovative Smart Grid Technologies - Asia (ISGT ASIA), Bangkok, 2015, pp. 1-6.

[7] T. Himeno and T. Ikegami, "Effects of home energy management systems for reduction of renewables output curtailment," 2015 IEEE Innovative Smart Grid Technologies - Asia (ISGT ASIA), Bangkok, 2015, pp. 1-6.

[8] S. W. Kim, J. W. Lee, J. Kim, Y. T. Yoon and Y. G. Jin, "Framework for determining the compensation price for output curtailment of distributed generation within active distribution network management," 2015 IEEE PES Asia-Pacific Power and Energy Engineering Conference (APPEEC), Brisbane, QLD, 2015, pp. 1-5.

[9] M. J. Dolan, E. M. Davidson, I. Kockar, G. W. Ault and S. D. J. McArthur, "Reducing Distributed Generator Curtailment Through Active Power Flow Management," in IEEE Transactions on Smart Grid, vol. 5, no. 1, pp. 149-157, Jan. 2014.

[10] D. Boldt, P. Faria and Z. Vale, "Study and analysis of wind curtailment situations and developing an appropriated methodology for its management," 2015 IEEE Eindhoven PowerTech, Eindhoven, 2015, pp. 1-6.

[11] Gesetz für den Ausbau Erneuerbarer Energien 2017, §8

[12] Gesetz für den Ausbau Erneuerbarer Energien 2017, §12

[13] Bundesnetzagentur, Referat 603, "Quartalsbericht zu Netz- und Systemsicherheitsmaßnahmen: Viertes Quartal und Gesamtjahr 2016“", Bundesnetzagentur, Bonn, p 6, May 2017

[14] Bundesamt für Sicherheit in der Informationstechnik, „Technische Richtlinie BSI TR-03109-1 Anforderungen an die Interoperabilität der Kommunikationseinheit eines intelligenten Messsystems", BSI, Bonn 2013, pp 52-57, 72-78.

[15] H2020 InterFlex Project [Online]. Available: http://interflex-h2020.com; [accessed 14.02.2018]

[16] The MathWorks, Inc., MATLAB and Simulink Sudent Suite 2017b, Natrick, Massachusetts, United States.

[17] J. Lofberg, "YALMIP: A toolbox for modeling and optimization in MATLAB," 2004 IEEE International Conference on Robotics and Automation, 2004.

[18] Inc. Gurobi Optimization, Gurobi Optimizer Reference Manual [Online]. Available: http://www.gurobi.com/ [accessed 28.03.2018] 